\title{
ARTICLE
}

Molecular Diagnostics

\section{Lactate concentration in breast cancer using advanced magnetic resonance spectroscopy}

\author{
Sai Man Cheung ${ }^{1}$, Ehab Husain ${ }^{2}$, Yazan Masannat ${ }^{3}$, lain D. Miller ${ }^{2}$, Klaus Wahle ${ }^{4}$, Steven D. Heys ${ }^{3}$ and Jiabao He ${ }^{1}$
}

BACKGROUND: Precision medicine in breast cancer demands markers sensitive to early treatment response. Aerobic glycolysis (AG) upregulates lactate dehydrogenase A (LDH-A) with elevated lactate production; however, existing approaches for lactate quantification are either invasive or impractical clinically.

METHODS: Thirty female patients (age 39-78 years, 15 grade II and 15 grade III) with invasive ductal carcinoma were enrolled. Lactate concentration was quantified from freshly excised whole tumours with double quantum filtered (DQF) magnetic resonance spectroscopy (MRS), and Nottingham Prognostic Index (NPI), LDH-A and proliferative marker Ki-67 were assessed histologically. RESULTS: There was a significantly higher lactate concentration $(t=2.2224, p=0.0349)$ in grade III $(7.7 \pm 2.9 \mathrm{mM})$ than in grade II $(5.5 \pm 2.4 \mathrm{mM})$. Lactate concentration was correlated with NPI $(\rho=0.3618, p=0.0495)$, but not with Ki-67 $(\rho=0.3041, p=0.1023)$ or tumour size $(r=0.1716, p=0.3645)$. Lactate concentration was negatively correlated with LDH-A $(\rho=-0.3734, p=0.0421)$. CONCLUSION: Our results showed that lactate concentration in whole breast tumour from DQF MRS is sensitive to tumour grades and patient prognosis.

British Journal of Cancer (2020) 123:261-267; https://doi.org/10.1038/s41416-020-0886-7

\section{BACKGROUND}

The 10-year survival rate of breast cancer has improved substantially from 40.1 to $78.4 \%$ in the last 40 years, ${ }^{1}$ primarily resulting from advancement in chemotherapy ${ }^{2}$ and targeted endocrine modulation. ${ }^{3}$ Non-responding patients not only are exposed to side effects and possible life threatening complications, ${ }^{4}$ but also may experience disease progression during treatment and delayed surgery, ${ }^{5}$ demanding an early response marker beyond crude tumour size. Aerobic glycolysis (AG), a central feature of tumour metabolism, fuels rapid growth with elevated glucose consumption and subsequent lactate production. ${ }^{6}$ Malignant transformation is underscored by genetic mutation, activating hypoxia inducible factor 1 (HIF-1) and subsequent upregulation of lactate dehydrogenase A (LDH-A), leading to increased conversion of pyruvate to lactate and the efflux of lactate through monocarboxylate transporter 4 (MCT4). ${ }^{7}$ The accumulation of lactate generates an acidic environment suppressing normal immunological functions while providing fuel to tumour cells, resulting in accelerated local invasion. ${ }^{8}$ The inhibition of lactate production induces tumour regression in cancer xenografts, underpinning the role of lactate concentration as a response marker. ${ }^{9}$

Biochemical approaches, although capable of lactate concentration quantification, are performed on excised tissue or biopsy samples with limited spatial extent. ${ }^{18}$ F-Fluorodeoxyglucose (FDG) positron emission tomography (PET) sensitive to glucose uptake, suffers from the dependency on glucose delivery and exposure of patients to ionising radiation. ${ }^{10}$ Hyperpolarised ${ }^{13} \mathrm{C}$-pyruvate magnetic resonance spectroscopic imaging maps the conversion of pyruvate to lactate, but suffers from the high cost of labelled pyruvate and specialist equipment. ${ }^{11}$ Conventional ${ }^{1} \mathrm{H}$ magnetic resonance spectroscopy (MRS) allows lactate quantification in neuro-oncology, ${ }^{12}$ but suffers from overwhelming contamination signal from lipid in breast. Double quantum filtered (DQF) MRS, a method recently available for clinical research, ${ }^{13}$ allows lactate quantification in high lipid tissue such as breast using standard hardware, but was so far only examined in proof of concept case studies. ${ }^{14}$

We therefore hypothesise that lactate concentration derived from DQF MRS is sensitive to tumour grades and is associated with prognosis.

\section{METHODS}

To probe the hypothesis, we conducted a two-group cross sectional study examining the lactate concentration of whole tumour freshly excised from patients, with further comparison against histopathological findings (Fig. 1).

Study approval

The study was approved by the North West-Greater Manchester East Research Ethics Committee (REC Reference: 16/NW/0032), and signed written informed consent was obtained from the patients prior to inclusion in the study.

\footnotetext{
${ }^{1}$ Institute of Medical Sciences, School of Medicine, University of Aberdeen, Aberdeen, UK; ${ }^{2}$ Pathology Department, Aberdeen Royal Infirmary, Aberdeen, UK; ${ }^{3}$ Breast Unit, Aberdeen Royal Infirmary, Aberdeen, UK and ${ }^{4}$ Strathclyde Institute of Pharmacy and Biological Sciences, University of Strathclyde, Glasgow, UK

Correspondence: Sai Man Cheung (g.cheung@abdn.ac.uk)

These authors contributed equally: Sai Man Cheung, Ehab Husain
}

Received: 5 February 2020 Revised: 9 April 2020 Accepted: 22 April 2020

Published online: 19 May 2020 


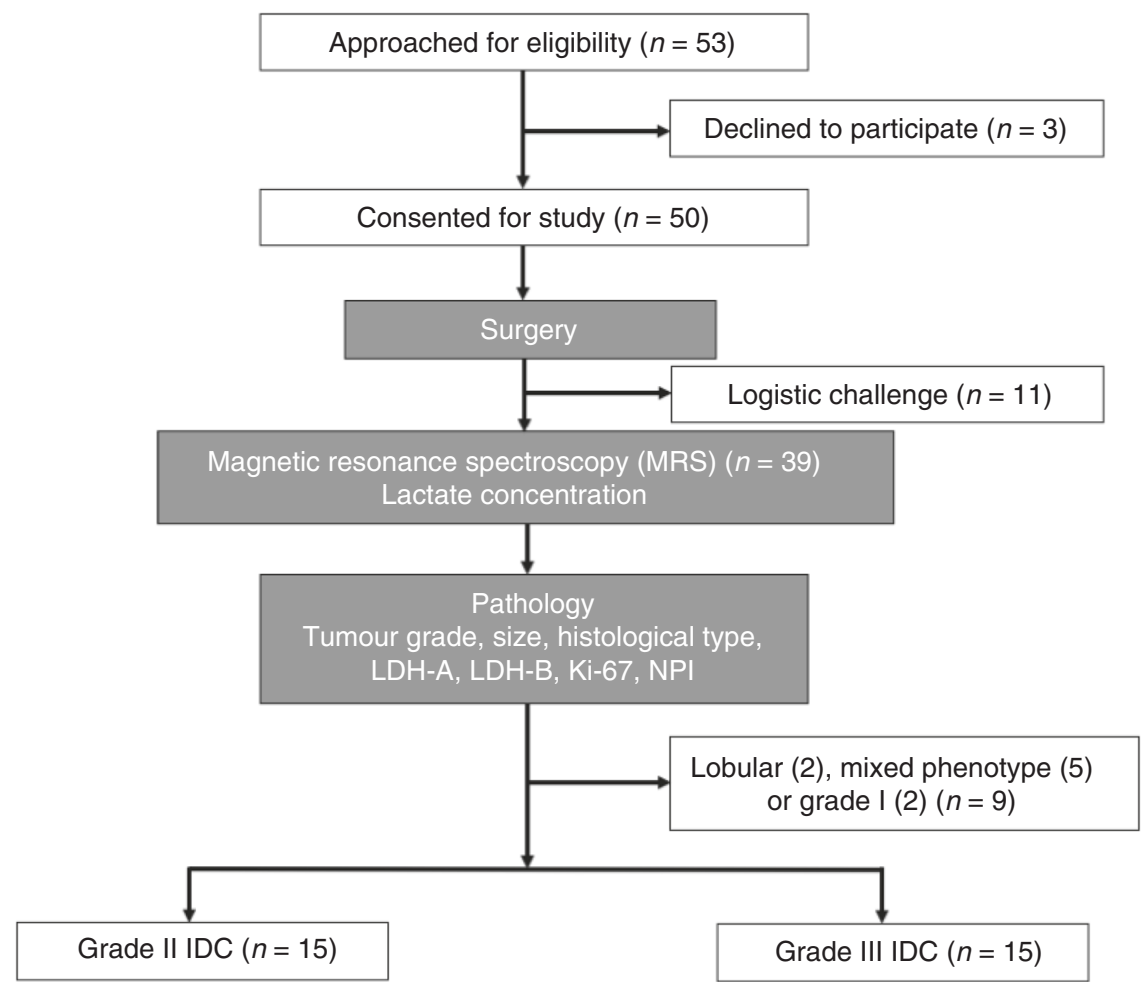

Fig. 1 Study design. The study adopted a two-group cross sectional arrangement as shown in this flow chart. Among 53 consecutive patients identified and approached, 50 patients were consented and received wide local excision or mastectomy. After surgery, the freshly excised tumours were scanned on a clinical 3 T MRI scanner to derive lactate concentration within the whole tumour using double quantum filtered (DQF) magnetic resonance spectroscopy (MRS). Subsequently, histopathological analysis provided tumour grade, size, histological type, lactate dehydrogenase A and B (LDH-A and LDH-B), Ki-67 expression and Nottingham Prognostic Index (NPI). Thirty patients with invasive ductal carcinoma (IDC) (15 grade II and 15 grade III) were eventually entered into the study and used for all statistical analysis.

\section{Patients}

Thirty female patients (age $39-78$ years, 15 grade II and 15 grade III) with invasive ductal carcinoma were enrolled in the study. Only patients undergoing wide local excision or mastectomy, and with a tumour size larger than $1 \mathrm{~cm}$ in diameter on ultrasound were eligible. Patients with previous breast malignancies or who had undergone prior neoadjuvant chemotherapy or neoadjuvant hormonal therapy were not eligible. In total, 50 patients consented to be included in the study from 53 consecutive patients in the Breast Unit at Aberdeen Royal Infirmary. From these 50 resected tumour specimens, 39 were scanned, with the remainder excluded because of either theatre delays or MRI scanner unavailability. Following histopathological examination of the excised tumour, alterations in tumour subtype or grade from biopsy that classified the tumour differently from grade II/III invasive ductal in type were excluded. In total, nine tumour specimens, including two lobular phenotype, five mixed phenotype and two grade I, were excluded.

Power calculation was based on Cohen's $d_{1}^{15}$ using the reported difference in lactate concentration of $2 \mathrm{mM}$ between aggressive and non-aggressive tumours in xenografted mice models, ${ }^{16}$ and standard deviation (SD) of $3 \mathrm{mM}$ determined from repeated phantom experiment. Therefore, to show a significant mean difference between groups at the 5\% level with $80 \%$ power using an independent sample design would require 15 subjects per group. ${ }^{17}$

\section{Magnetic resonance}

After wide local excision or mastectomy, the fresh tissue (without formalin treatment) was immediately transported to the Aberdeen Biomedical Imaging Centre (transportation time less than 5 min, Fig. 1). The ex vivo specimen was scanned in air and positioned in the isocentre of the scanner. The data were acquired on a $3 \mathrm{~T}$ whole-body clinical MRI scanner (Achieva TX, software version R5.1.7, Philips Healthcare, Best, Netherlands) using a body coil for uniform transmission and a 32-channel receiver head coil for high sensitivity detection. The DQF sequence was implemented locally using Philips Pulse Programming Environment (PPE). The $\mathrm{T}_{1}$-weighted anatomical images were acquired using standard $3 \mathrm{D}$ sequence with an isotropic voxel size of $1 \mathrm{~mm}$, matrix size of $220 \times 220$, repetition time (TR) of $5.2 \mathrm{~ms}$, echo time (TE) of $2.7 \mathrm{~ms}$, imaging volume encompassing the specimen. Subsequently, the lactate spectrum was acquired from a single voxel snug-fit to the tumour using single voxel DQF PRESS sequence, ${ }^{13}$ with TR/TE of $1.25 \mathrm{~s} / 144 \mathrm{~ms}$, spectral editing frequency at $4.1 \mathrm{ppm}$ and 512 averages. A reference spectrum was acquired from the same voxel using single voxel PRESS sequence with TR/TE of $1.25 \mathrm{~s} / 144 \mathrm{~ms}$, 16 averages. The total acquisition time for lactate spectrum was $11 \mathrm{~min}$.

All spectra were processed following standard procedures and quantified using AMARES algorithm ${ }^{18}$ in the jMRUI software (v3.0, TRANSACT, Leuven, Belgium). ${ }^{19}$ Water and lactate amplitudes were quantified from reference and lactate spectra, respectively, using corresponding prior knowledge databases. ${ }^{20}$ The lactate concentration in the tumour was then computed from water and lactate amplitudes, literature breast tissue biochemical composition and relaxation properties on the $3 \mathrm{~T}$ scanner. ${ }^{21}$ The quantification of lactate concentration was conducted following written procedure and blinded from group allocation. Full details of quantification and spectra from breast tumour specimens are provided in Supplementary Information. 
Table 1. Patient demography.

\begin{tabular}{|c|c|c|c|c|}
\hline Characteristic & All $(n=30)$ & Grade II $(n=15)$ & Grade III $(n=15)$ & $p$-value \\
\hline Age & $61.1 \pm 11.5$ & $61.0 \pm 12.0$ & $61.2 \pm 11.3$ & 0.9628 \\
\hline Body mass index (BMI) & $30.4 \pm 6.4$ & $30.4 \pm 6.6$ & $30.3 \pm 6.6$ & 0.9771 \\
\hline Tumour size $(\mathrm{cm})$ & $2.5 \pm 0.8$ & $2.4 \pm 0.9$ & $2.5 \pm 0.7$ & 0.7650 \\
\hline Tumour volume $\left(\mathrm{cm}^{3}\right)^{a}$ & $20.0 \pm 21.3$ & $20.3 \pm 19.2$ & $19.6 \pm 23.9$ & 0.9335 \\
\hline Nottingham prognostic index (NPI) & $4.41(3.62-4.56)$ & $3.62(3.46-4.29)$ & $4.50(4.44-5.02)$ & $0.0001^{*}$ \\
\hline \multicolumn{5}{|l|}{ Tumour type } \\
\hline \multicolumn{5}{|l|}{ pTNM Stage } \\
\hline 1 & 5 & 3 & 2 & 1.000 \\
\hline II & 25 & 12 & 13 & \\
\hline Lymphovascular invasion (LVI) & 17 & 9 & 8 & 1.000 \\
\hline Lymph node involvement & 8 & 4 & 4 & 1.000 \\
\hline Oestrogen receptor $(\mathrm{ER}+)$ & 22 & 13 & 9 & 0.215 \\
\hline Human epidermal growth factor receptor 2 (HER2+) & 6 & 2 & 4 & 0.651 \\
\hline Triple-negative breast cancer (TNBC) & 7 & 1 & 6 & 0.080 \\
\hline
\end{tabular}

Patient demography and clinical histopathological findings of excised breast tumours are shown for each group and the entire cohort. Quantitative data are expressed as mean and standard deviation (apart from Nottingham Prognostic Index where median and interquartile range are shown), while qualitative data expressed as number of positive cases.

*Significant findings $(p<0.05)$ are marked.

${ }^{\mathrm{a}}$ Tumour volume extracted from $\mathrm{T}_{2}$-weighted MRI.

Histopathological analysis

Upon the completion of scanning, the specimen was immediately transported to the Pathology Department at Aberdeen Royal Infirmary (transportation time less than five minutes, Fig. 1) for formalin fixation. Routine clinical histopathological examination was undertaken on the specimen to define the tumour characteristics (including size and grade) along with the nodal status for Nottingham Prognostic Index (NPI). ${ }^{22}$ Subsequently, immunostaining was performed in a single batch for $\mathrm{Ki}-67,{ }^{23} \mathrm{LDH}-\mathrm{A}^{24}$ and $\mathrm{LDH}-\mathrm{B}^{25}$ including appropriate positive controls. The immunostains were assessed semi-quantitatively by two pathologists independently (EH and IM), blinded from group allocation.

Statistical analysis

All statistical analysis was performed in the SPSS software (Release 23.0, SPSS Inc., Chicago, IL, USA). Normality was determined on all the collected data using the Shapiro-Wilk test. Two-sample $t$-tests were performed on normally distributed data to assess difference between tumours with grade II and grade III histology, and MannWhitney $U$ tests for non-normally distributed data. Fisher's exact tests were applied to study the association between grades and other tumour pathological features. Pearson's correlation test was performed between lactate concentration and tumour size, with Spearman's rank correlation tests for lactate concentration against NPI, Ki-67 and LDH-A. A p-value $<0.05$ was considered statistically significant.

\section{RESULTS}

The patient characteristics are shown in Table 1. All patient demographics, apart from NPI, were normally distributed. There were no significant differences in age, body mass index and tumour size between groups. Lactate was measurable in all spectra, defined as Cramér-Rao lower bound (CRLB) of the fit below 30\%.

There was a significantly higher $(t=2.2224, p=0.0349)$ lactate concentration in grade III $(7.7 \pm 2.9 \mathrm{mM})$ than in grade II $(5.5 \pm$ $2.4 \mathrm{mM}$ ) (Table 2, Fig. 2a). NPI was significantly higher $(z=3.819, p$ $=0.0001$ ) in grade III (median: 4.50; interquartile range (IQR): 4.44-5.02) than in grade II (median: 3.46; IQR: 3.46-4.29) (Table 1, Fig. 2b). There was a significantly higher $(z=3.712, p=0.0002)$ Ki-67 expression in grade III (median: 22.9\%, IQR: 16.7-40.2\%) than in grade II (median: $9.7 \%$, IQR: 6.3-16.4\%) (Table 2, Fig. 2c). There was a significantly higher $(z=2.743, p=0.0061) \mathrm{LDH}-\mathrm{A}$ expression in grade II (median: 300, IQR: 250-300) than in grade III (median: 100, IQR: 80-250) (Table 2, Fig. 2d). There was a significantly higher $(z=2.821, p=0.0048)$ LDH-B expression in grade III (median: 40, IQR: 0-120) than in grade II (median: 0, IQF: 0-10) (Table 2).

There was a significant correlation between lactate concentration and NPI ( $\rho=0.3618, p=0.0495$, Table 2, Fig. 3a). There were no significant correlations between lactate concentration against Ki-67 expression $(\rho=0.3041, p=0.1023$, Table 2, Fig. $3 b)$ or tumour size $(r=0.1716, p=0.3645$, Fig. 3c). There was a significant negative correlation between lactate concentration and LDH-A ( $\rho=-0.3734, p=0.0421$, Table 2, Fig. 3d).

\section{DISCUSSION}

In this work, we found lactate concentration was significantly higher in grade III breast tumours compared to grade II. Increased lactate concentration was associated with worse NPI, not associated with tumour proliferative activity or tumour size, and negatively associated with LDH-A expression. Hence, lactate concentration derived from DQF MRS is a marker sensitive to tumour grades, with an association to prognosis.

Lactate concentration, observed in this study, is in agreement with findings in breast cancer cell culture, ${ }^{26}$ xenografted animal tumour models, ${ }^{16}$ ex vivo human brain tumours ${ }^{27}$ and brain tumours in patients. ${ }^{12}$ Since accumulated lactate alters the cellular physical appearance through the inflammatory activities and deregulation of immune function, ${ }^{8}$ the sensitivity to tumour grade is through the sub-scores associated with cellular appearance of tubule formation, nuclear pleomorphism and mitoses. Lactate concentration quantified over the entire tumour addresses the drawbacks in biopsy methods of intratumoural heterogeneity and partial sampling error. Lactate concentration is a quantitative objective measure compared to immunostaining methods and FDG-PET, reducing the demand on manpower and ambiguity. Hence, lactate concentration from DQF MRS is a non-invasive marker sensitive to tumour grade, with significant advantages over current methods for quantifying $A G$.

The correlation between lactate concentration and NPI indicates the prognostic value of lactate concentration in breast cancer, in 


\begin{tabular}{|c|c|c|c|c|c|c|}
\hline & 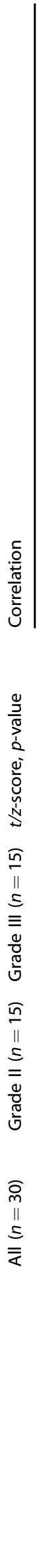 & 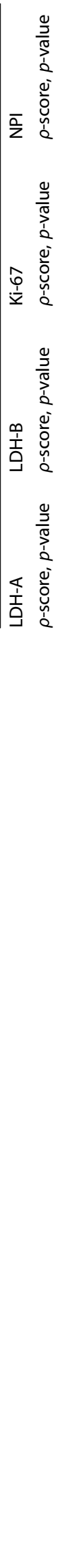 & 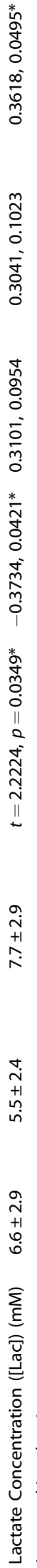 & 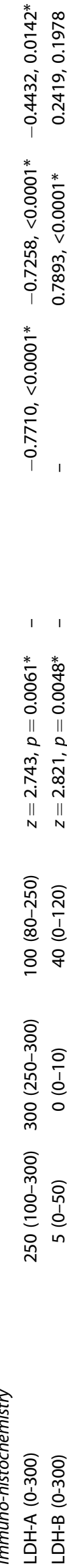 & 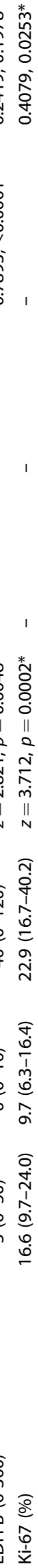 & 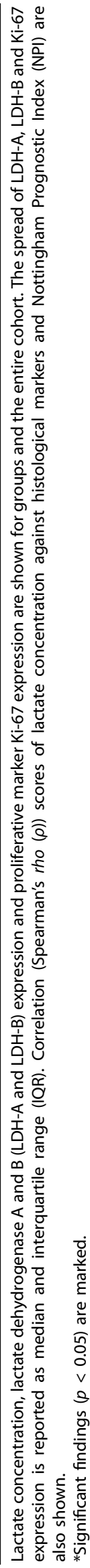 \\
\hline
\end{tabular}

line with elevated release of lactate in highly malignant breast cancer cells. ${ }^{26}$ Lactate supports advancing epithelial cancer cells as energy source, ${ }^{6}$ while macrophage function is severely compromised at high lactate concentration $(>15 \mathrm{mM})$ with diminished production of natural killer cells and apoptosis of T cells. ${ }^{8}$ Lactate concentration does not show significant correlation with crude tumour size or Ki-67, underscoring lactate concentration as a specific marker to AG. Proliferative activity marker Ki-67 reveals the rate of division of cellular nuclear materials ${ }^{28}$ (that is possibly discordant with the mitotic index ${ }^{29}$ ), while lactate enhances the survival advantage of tumour cells reflecting invasion capability. ${ }^{6}$ The negative correlation against LDH-A was likely the result of feedback inhibition ${ }^{30}$ and depletion of pyruvate and nicotinamide adenine dinucleotide hydrogen $(\mathrm{NADH})^{31}$ in higher grade tumour. The observed higher expression of LDH-A has been previously found in less aggressive 67NR breast cancer cells under hypoxia in preclinical model ${ }^{16}$ and more differentiated tumours in patients with gastric cancer, ${ }^{32}$ while observed higher LDH-B in higher grade is in agreement with previous studies in breast cancer. ${ }^{33}$ However, carbonic anhydrase IX (CA9), a strong prognostic marker, has been recently shown to induce an acidic tumour extracellular $\mathrm{pH}$ in vivo, ${ }^{34}$ decoupling lactate production regulated by LDH and lactate concentration. Hence, lactate concentration is a sensitive prognostic marker, specific to the invasion capability resulted from an acidic extracellular environment.

The lactate concentration obtained from single voxel approach is an average over the whole tumour, and does not inform the spatial distribution of lactate within tumour, limiting the accuracy in the cases with large disparity between tumour core and rim. $^{26}$ Although triple-negative breast cancer is postulated to be more aggressive, there is no significant difference in lactate concentration between triple-negative (6 cases, mean: $8.4 \mathrm{mM}$ ) and non-triple-negative (nine cases, mean: $7.2 \mathrm{mM}$ ) in our grade III cohort, indicating no proven relation to this potential confounding factor. The deterioration of tissue after excision may affect the accuracy of lactate concentration quantification; however, the median time gap from excision to the middle of the DQF MRS scan was 25 min, significantly below the time frame for observing change in lactate concentration in postmortem tissues $(3 \mathrm{~h}) .^{35}$ The freshly excised tumour allows the use of a high sensitivity receiver head coil for improved measurement accuracy, while eliminating biological noise. Restriction of tumours to grade II and III with a size larger than $1 \mathrm{~cm}(24$ specimens $>2 \mathrm{~cm}$ ) is to represent patient population likely receiving neoadjuvant therapy. The restriction of phenotype to invasive ductal carcinoma, the most common breast cancer, eliminates phenotype as a confounding factor, allowing adequate statistical power in a small patient cohort. The sample size is small; however, careful power calculation was conducted to assess the appropriate sample size $^{16}$ before the evaluation of the lactate concentration from ex vivo tumours.

The current acquisition of lactate spectrum was long (11 min) and only suitable for lactate concentrations higher than $1.5 \mathrm{mM}$, demanding future development work to improve the sensitivity. Lactate was measurable in all spectra, and quantification was limited to lactate spectra with CRLB below $30 \%$. The overlap of the distribution in lactate concentration between groups limits the prognostic value of lactate concentration obtained using current methodology in breast cancer. MRS is well known to have high specificity but low sensitivity. Future technological improvement including digital hardware and adaptive signal processing for phased array coils ${ }^{36}$ should be incorporated in further development to improve prognostic value. Future longitudinal studies on patients undergoing neoadjuvant chemotherapy will confirm the prognostic and treatment monitoring value of lactate concentration, and multicentre study with larger cohort size covering a complete range of phenotypes is essential for clinical adoption. 
a

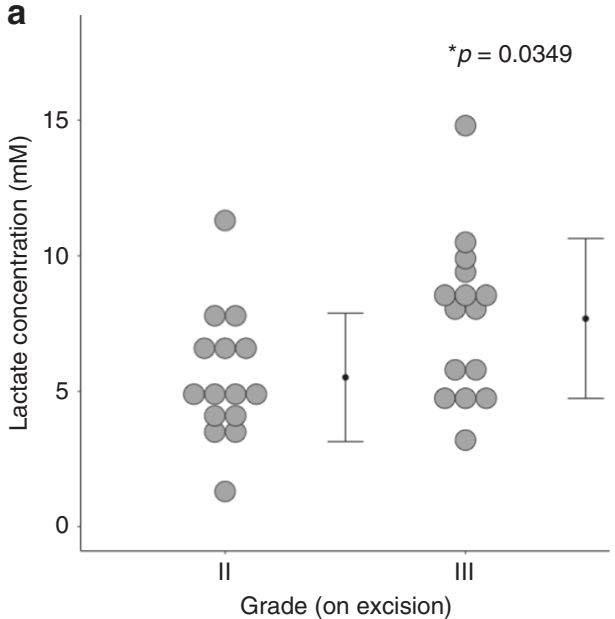

C

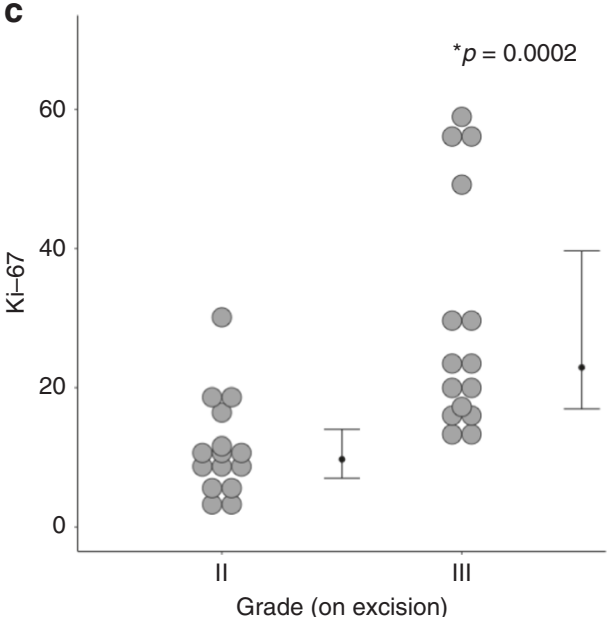

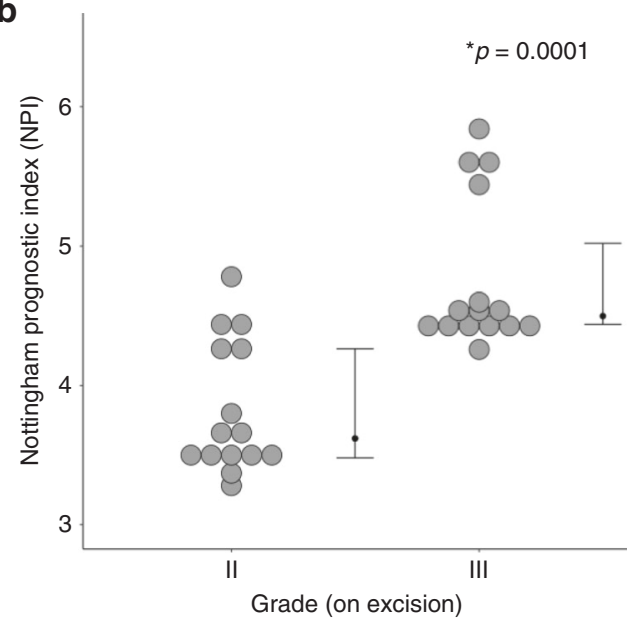

d

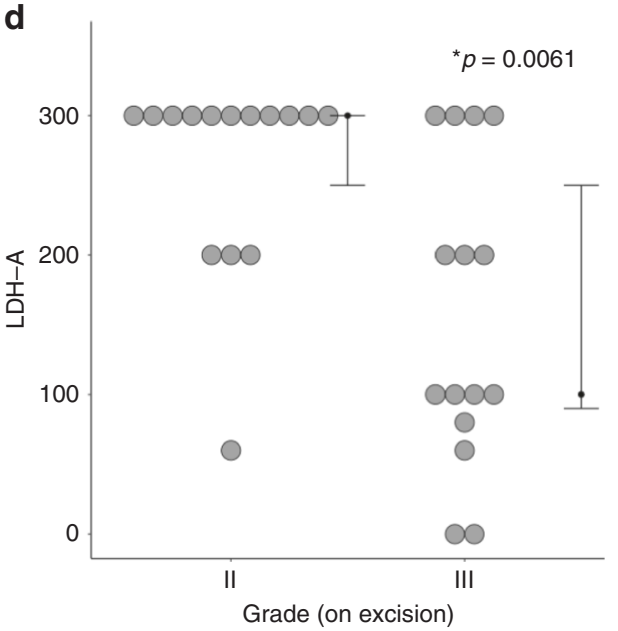

Fig. 2 Lactate concentration, NPI and histological markers Ki-67 and LDH-A between grade II and III breast tumours. a Lactate concentration $(n=15)$, b Nottingham Prognostic Index (NPI) $(n=15)$, c Ki-67 $(n=15)$ and d lactate dehydrogenase A (LDH-A) expression ( $n=$ 15). Each dot represents the measurement obtained in each patient, and the dots are organised in two columns corresponding to the tumour grades. For lactate concentration, the error bar indicates the mean and standard deviation. For NPI, Ki-67 and LDH-A expression, it indicates the median and interquartile range. The two-tailed Student's $t$-test was performed between the groups for lactate concentration, while MannWhitney $U$ tests for NPI, Ki-67 and LDH-A expression. $P<0.05$ was considered statistically significant and is marked by 'asterisk'.

Lactate concentration from DQF MRS is a non-invasive marker sensitive to breast tumour grade and is associated with NPI as demonstrated in a cross sectional study. Lactate concentration provides a valuable clinical research tool for potential identification of treatment targets and application to improve image guided neoadjuvant chemotherapy.

\section{ACKNOWLEDGEMENTS}

We would like to thank Dr. Nicholas Senn for conducting data auditing, Dr. Matthew Clemence (Philips Healthcare Clinical Science, UK) for clinical scientist support, Dr. Tim Smith for biologist support, Mr. Gordon Buchan for technician support, Ms Bolanle Brikinns for patient recruitment support, Ms Dawn Younie for logistic support, Prof. Andrew M. Blamire for advice on MRS. We would also like to thank Mr Roger Bourne and Ms Mairi Fuller for providing access to the patients.

\section{AUTHOR CONTRIBUTIONS}

S.C. managed study paperwork, collected the data, analysed the data, performed statistical analysis, interpreted the results and drafted the manuscript. E.H. secured the funding, designed the study, performed histopathological analysis, interpreted the results and reviewed the manuscript. Y.M. recruited the patients, performed the surgical intervention, interpreted the results and reviewed the manuscript. I.M. conducted histopathological analysis and reviewed the manuscript. K.W. interpreted the results and reviewed the manuscript. S.H. secured the funding, designed the study, interpreted the results and reviewed the manuscript. J.H. secured the funding, designed the study, coordinated the experiments, collected the data, interpreted the results and drafted the manuscript.

\section{ADDITIONAL INFORMATION}

Ethics approval and consent to participate The study was done in accordance with the Declaration of Helsinki. The study was approved by the North West-Greater Manchester East Research Ethics Committee (REC Reference: 16/NW/0032), and signed written informed consent was obtained from the patients prior to inclusion in the study.

Data availability Data supporting this publication are stored at Institute of Medical Sciences and available upon request.

Competing interests The authors declare no competing interests.

Funding information This project was funded by Friends of Aberdeen and North Centre for Haematology, Oncology and Radiotherapy (ANCHOR) (RS2015 004). Sai Man Cheung's PhD study was jointly supported by Elphinstone scholarship, Roland Sutton Academic Trust and John Mallard scholarship. 

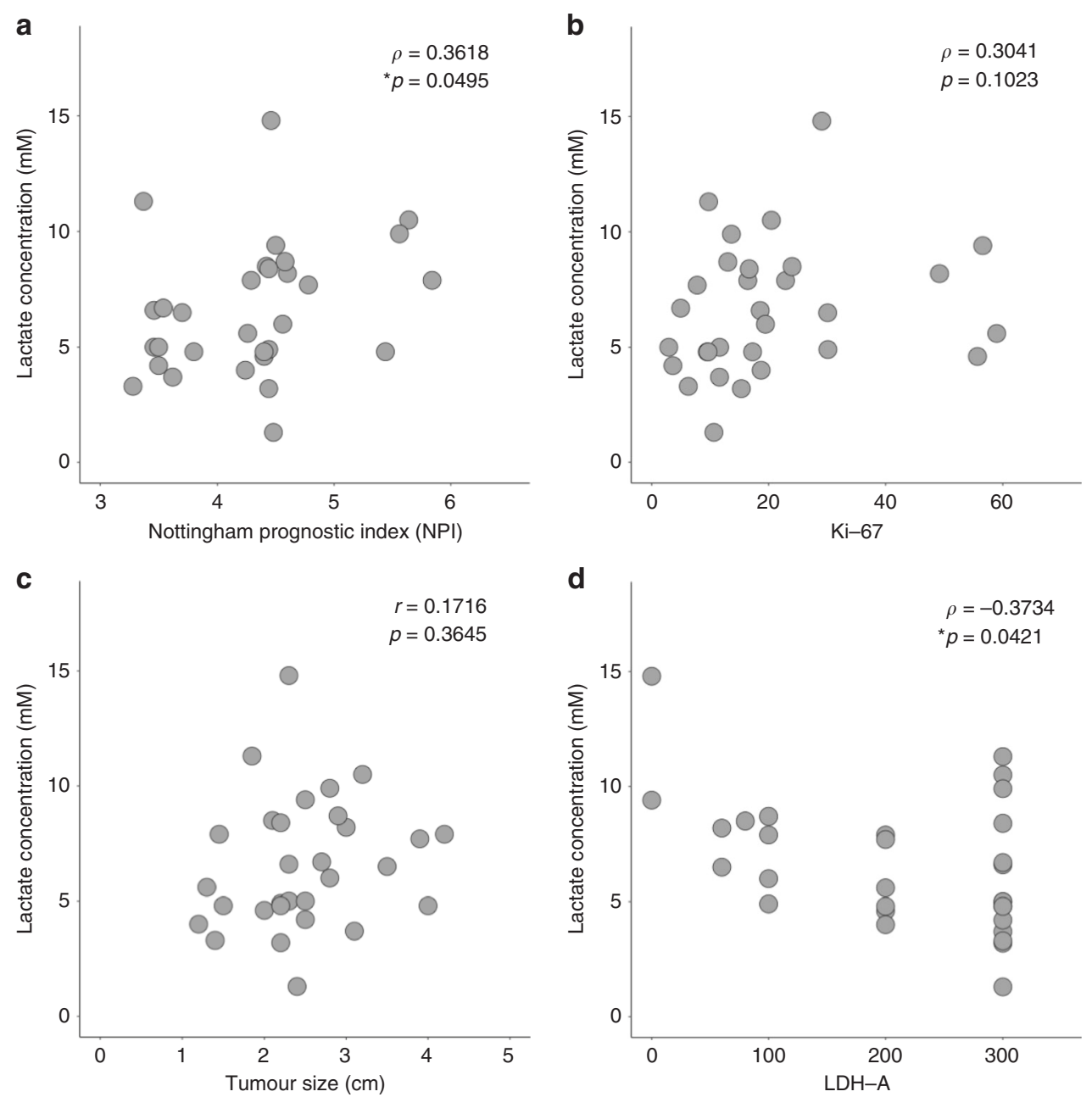

Fig. 3 Association between lactate concentration and NPI, Ki-67, tumour size and LDH-A. Lactate concentration was correlated against a Nottingham Prognostic Index (NPI) $(n=30)$, b Ki-67 expression $(n=30)$, c tumour size $(n=30)$ and d lactate dehydrogenase A (LDH-A) expression $(n=30)$ within the entire cohort and shown as scatter plots. Spearman's correlation tests ( $\rho$ score: $\mathbf{a}, \mathbf{b}$, d), Pearson's correlation test ( $r$ score: $c)$ were used and corresponding $p$-values are displayed. Statistically significant $p$-values $(<0.05)$ are marked by 'asterisk'.

Supplementary information is available for this paper at https://doi.org/10.1038/ s41416-020-0886-7.

Publisher's note Springer Nature remains neutral with regard to jurisdictional claims in published maps and institutional affiliations.

\section{REFERENCES}

1. Quaresma, M., Coleman, M. \& Rachet, B. 40-year trends in an index of survival for all cancers combined and survival adjusted for age and sex for each cancer in England and Wales, 1971-2011: a population-based study. Lancet 385, 1206-1218 (2015).

2. Smith, I. C., Heys, S. D., Hutcheon, A. W., Miller, I. D., Payne, S., Gilbert, F. J. et al. Neoadjuvant chemotherapy in breast cancer: significantly enhanced response with docetaxel. J. Clin. Oncol. 20, 1456-1466 (2002).

3. Burstein, H. J., Prestrud, A. A., Seidenfeld, J., Anderson, H., Buchholz, T. A., Davidson, N. E. et al. American Society of Clinical Oncology clinical practice guideline: update on adjuvant endocrine therapy for women with hormone receptor-positive breast cancer. J. Clin. Oncol. 28, 3784-3796 (2010).

4. Redana, S., Sharp, A., Lote, H., Mohammed, K., Papadimitraki, E., Capelan, M. et al. Rates of major complications during neoadjuvant and adjuvant chemotherapy for early breast cancer: an off study population. Breast 30, 13-18 (2016).
5. Cain, H., Macpherson, I. R., Beresford, M., Pinder, S. E., Pong, J. \& Dixon, J. M. Neoadjuvant therapy in early breast cancer: treatment considerations and common debates in practice. Clin. Oncol. 29, 642-652 (2017).

6. Vander Heiden, M. G., Cantley, L. C. \& Thompson, C. B. Understanding the Warburg effect: the metabolic requirements of cell proliferation. Science 324, 1029-1033 (2009).

7. Ullah, M. S., Davies, A. J. \& Halestrap, A. P. The plasma membrane lactate transporter MCT4, but not MCT1, is up-regulated by hypoxia through a HIF-1adependent mechanism. J. Biol. Chem. 281, 9030-9037 (2006).

8. Brand, A., Singer, K., Koehl, G. E., Kolitzus, M., Schoenhammer, G., Thiel, A. et al. LDHA-associated lactic acid production blunts tumor immunosurveillance by $T$ and NK cells. Cell Metab. 24, 657-671 (2016).

9. Farabegoli, F., Vettraino, M., Manerba, M., Fiume, L., Roberti, M. \& Di Stefano, G. Galloflavin, a new lactate dehydrogenase inhibitor, induces the death of human breast cancer cells with different glycolytic attitude by affecting distinct signaling pathways. Eur. J. Pharm. Sci. 47, 729-738 (2012).

10. Avril, N., Menzel, M., Dose, J., Schelling, M., Weber, W., Jänicke, F. et al. Glucose metabolism of breast cancer assessed by ${ }^{18}$ F-FDG PET: histologic and immunohistochemical tissue analysis. J. Nucl. Med. 42, 9-16 (2001).

11. Adamson, E. B., Ludwig, K. D., Mummy, D. G. \& Fain, S. B. Magnetic resonance imaging with hyperpolarized agents: methods and applications. Phys. Med. Biol. 62, R81-R123 (2017).

12. Howe, F. A., Barton, S. J., Cudlip, S. A., Stubbs, M., Saunders, D. E., Murphy, M. et al. Metabolic profiles of human brain tumors using quantitative in vivo ${ }^{1} \mathrm{H}$ magnetic resonance spectroscopy. Magn. Reson. Med. 49, 223-232 (2003).

13. Meyerspeer, M., Kemp, G. J., Mlynárik, V., Krššák, M., Szendroedi, J., Nowotny, P. et al. Direct noninvasive quantification of lactate and high energy phosphates 
simultaneously in exercising human skeletal muscle by localized magnetic resonance spectroscopy. Magn. Reson. Med. 57, 654-660 (2007).

14. He, Q. H., Shungu, D. C., van Zijl, P. C. M., Bhujwalla, Z. M. \& Glickson, J. D. Singlescan in vivo lactate editing with complete lipid and water suppression by selective multiple-quantum-coherence transfer (Sel-MQC) with application to tumors. J. Magn. Reson. Ser. B 106, 203-211 (1995).

15. Cohen, J. A power primer. Psychol. Bull. 112, 155-159 (1992).

16. Serganova, I., Rizwan, A., Ni, X., Thakur, S. B., Vider, J., Russell, J. et al. Metabolic imaging: a link between lactate dehydrogenase $A$, lactate, and tumor phenotype. Clin. Cancer Res. 17, 6250-6261 (2011)

17. Wendorf, C. A. Statistics for psychologists Part V: statistical tables, pp 1-25, University of Wisconsin (2014).

18. Vanhamme, L. \& van den Boogaart, A. Improved method for accurate and efficient quantification of MRS data with use of prior knowledge. J. Magn. Reson. 129, 35-43 (1997).

19. Naressi, A., Couturier, C., Castang, I., de Beer, R. \& Graveron-Demilly, D. Java-based graphical user interface for MRUI, a software package for quantitation of in vivo/medical magnetic resonance spectroscopy signals. Comput. Biol. Med. 31, 269-286 (2001)

20. Payne, G. S., Harris, L. M., Cairns, G. S., Messiou, C., deSouze, N. M., Macdonald, A. et al. Validating a robust double-quantum-filtered ${ }^{1} \mathrm{H}$ MRS lactate measurement method in high-grade brain tumours. NMR Biomed. 29, 1420-1426 (2016).

21. Sijens, P. E., Dorrius, M. D., Kappert, P., Baron, P., Pijnappel, R. M. \& Oudkerk, M. Quantitative multivoxel proton chemical shift imaging of the breast. Magn. Reson. Imag. 28, 314-319 (2010).

22. Elston, C. W. \& Ellis, I. O. Pathological prognostic factors in breast cancer. I. The value of histological grade in breast cancer: experience from a large study with long-term follow-up. Histopathology 19, 403-410 (1991).

23. Tuominen, V. J., Ruotoistenmäki, S., Viitanen, A., Jumppanen, M. \& Isola, J. ImmunoRatio: a publicly available web application for quantitative image analysis of estrogen receptor (ER), progesterone receptor (PR), and Ki-67. Breast Cancer Res. 12, R56 (2010).

24. Dong, T., Liu, Z., Xuan, Q., Wang, Z., Ma, W. \& Zhang, Q. Tumor LDH-A expression and serum LDH status are two metabolic predictors for triple negative breast cancer brain metastasis. Sci. Rep. 7, 6069 (2017).

25. Dennison, J. B., Molina, J. R., Mitra, S., González-Angulo, A. M., Balko, J. M., Kuba, M. G. et al. Lactate dehydrogenase B: a metabolic marker of response to neoadjuvant chemotherapy in breast cancer. Clin. Cancer Res. 19, 3703-3713 (2013).

26. Rizwan, A., Serganova, I., Khanin, R., Karabeber, H., Ni, X., Thakur, S. et al. Relationships between LDH-A, lactate, and metastases in 4T1 breast tumors. Clin Cancer Res. 19, 5158-5169 (2013).

27. Wright, A. J., Fellows, G. A., Griffiths, J. R., Wilson, M., Bell, B. A. \& Howe, F. A. Ex-vivo HRMAS of adult brain tumours: metabolite quantification and assignment of tumour biomarkers. Mol. Cancer 9, 66 (2010).
28. Yerushalmi, R., Woods, R., Ravdin, P. M., Hayes, M. M. \& Gelmon, K. A. Ki67 in breast cancer: prognostic and predictive potential. Lancet Oncol. 11, 174-183 (2010).

29. Rossi, L., Laas, E., Mallon, P., Vincent-Salomon, A., Guinebretiere, J.-M., Lerebours, F. et al. Prognostic impact of discrepant $\mathrm{Ki} 67$ and mitotic index on hormone receptor-positive, HER2-negative breast carcinoma. Br. J. Cancer 113, 996-1002 (2015).

30. Valvona, C. J., Fillmore, H. L., Nunn, P. B. \& Pilkington, G. J. The regulation and function of lactate dehydrogenase $A$ : therapeutic potential in brain tumor. Brain Pathol. 26, 3-17 (2015).

31. Spriet, L., Howlett, R. \& Heigenhauser, G. An enzymatic approach to lactate production in human skeletal muscle during exercise. Med. Sci. Sports Exerc. 32, 756-763 (2000).

32. Sun, X., Sun, Z., Zhu, Z., Guan, H., Zhang, J., Zhang, Y. et al. Clinicopathological significance and prognostic value of lactate dehydrogenase $A$ expression in gastric cancer patients. PLOS ONE 9, e91068-e91069 (2014).

33. Mack, N., Mazzio, E. A., Bauer, D., Flores-Rozas, H. \& Soliman, K. F. A. Stable shRNA silencing of lactate dehydrogenase $A$ (LDHA) in human MDA-MB-231 breast cancer cells fails to alter lactic acid production, glycolytic activity, ATP or survival. Anticancer Res. 37, 1205-1212 (2017).

34. Lee, S.-H., McIntyre, D., Honess, D., Hulikova, A., Pacheco-Torres, J., Cerdán, S. et al. Carbonic anhydrase IX is a pH-stat that sets an acidic tumour extracellular $\mathrm{pH}$ in vivo. Br. J. Cancer 119, 622-630 (2018).

35. Donaldson, A. E. \& Lamont, I. L. Biochemistry changes that occur after death potential markers for determining post-mortem interval. PLOS ONE 8, e82011-10 (2013).

36. Mallikourti, V., Cheung, S. M., Gagliardi, T., Masannat, Y., Heys, S. D. \& He, J. Optimal phased-array signal combination for polyunsaturated fatty acids measurement in breast cancer using multiple quantum coherence MR spectroscopy at 3T. Sci. Rep. 9, 9259 (2019).

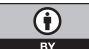

Open Access This article is licensed under a Creative Commons Attribution 4.0 International License, which permits use, sharing, adaptation, distribution and reproduction in any medium or format, as long as you give appropriate credit to the original author(s) and the source, provide a link to the Creative Commons license, and indicate if changes were made. The images or other third party material in this article are included in the article's Creative Commons license, unless indicated otherwise in a credit line to the material. If material is not included in the article's Creative Commons license and your intended use is not permitted by statutory regulation or exceeds the permitted use, you will need to obtain permission directly from the copyright holder. To view a copy of this license, visit http://creativecommons. org/licenses/by/4.0/.

(c) The Author(s) 2020 